Check for updates

Cite this: RSC Adv., 2017, 7, 50121

\title{
Intuitionistic study on the critical decomposition energy of ammonium perchlorate by SEM
}

\begin{abstract}
Jie Liu, ${ }^{*}$ Xiang Ke, (D) Ga-zi Hao, Lei Xiao, Wei Jiang and Feng-sheng Li
The decomposition course of Ammonium Perchlorate (AP) particles under electron energy is observed using a G2 pro Desktop Scanning Electron Microscope. The state that the AP particle is starting to decompose exhibiting cracks on its surface is observed and recorded using the S-4800II Fielding Emission Scanning Electron Microscope (FESEM), the Image Pro Plus (IPP) System is employed to obtain the projected area of that decomposed AP particle in the FESEM image, and the critical decomposition energy of the AP particle is calculated. Results have shown that the critical decomposition energy is decreased with the reduction of AP particle size. Especially when the AP particle size is approximately under $20 \mu \mathrm{m}$, the critical decomposition energy is sharply decreased, and the critical decomposition energy is very small if under $3 \mu \mathrm{m}$. This result is very helpful to explain why the burning rate of propellants and the brisance of Fuel Air Explosives (FAE) are enhanced and the sensitivities of those AP-based energetic materials are increased with the reduction of AP particle size. Furthermore, this method can be used to calculate in an intuitionistic way the critical decomposition energies of other crystalline materials.
\end{abstract}

Received 11th September 2017 Accepted 17th October 2017

DOI: $10.1039 / \mathrm{c} 7 \mathrm{ra10033g}$

rsc.li/rsc-advances objective reality. That is, logic and mathematics are not considered analytic activities wherein deep properties of objective reality are revealed and applied but are instead considered the application of internally consistent methods used to realize more complex mental constructs, regardless of their possible independent existence in an objective reality.

In this work, the S-4800II Field Emission Scanning Electron Microscope (FESEM) is employed to intuitionisticly research the critical decomposition energy of AP particles. The electron energy is emitted by the FESEM and inflicted on the AP particles, the critical decomposition state is observed and recorded by the FESEM, and the critical decomposition energy is calculated. The result has shown that the critical decomposition energy of AP particle is decreased with the reduction of the particle size, especially when the particle size is approximately under $20 \mu \mathrm{m}$, the critical decomposition energy is apparently decreased, and the critical decomposition energy is very small if under $3 \mu \mathrm{m}$. It is distinctly claimed that the superfine AP particles will be easily initiated to decompose by very small energy, so the burning rate or brisance of the AP-based energetic materials will be enhanced, and at the same time, their sensitivities to friction, impact and shock will be increased. Furthermore, it is potentially expectable to get the critical decomposition energies of other crystalline materials based on this method.

\section{Experimental}

\subsection{Materials and instruments}

The decomposition course of AP particles with an average size of $2.099 \mu \mathrm{m}$ is observed using the Desktop SEM (G2 pro Desktop
National Special Superfine Powder Engineering Research Center of China, School of Chemical Engineering, Nanjing University of Science and Technology, Nanjing, 210094, P. R. China. E-mail: jie_liu_njust@126.com; Tel: +86-025-84315042 
Scanning Electron Microscope). The AP particles with the size of $6 \mu \mathrm{m}$ are selected as an example how to observe the critical decomposition state and calculate the critical decomposition energy. The critical decomposition energies of other five kinds of AP particles, $1 \mu \mathrm{m}, 6 \mu \mathrm{m}, 10 \mu \mathrm{m}, 20 \mu \mathrm{m}, 60 \mu \mathrm{m}$ and $80 \mu \mathrm{m}$, are calculated. The AP particles selected to calculate the critical decomposition energy should be quasi-spherical or with regular shape. All of the researched AP samples are produced by Dalian North Chlorate Company of China.

\subsection{Observation of decomposition course}

The decomposition course of AP particles is observed using the Desktop SEM. The states, that the AP particles are separately affected by the electron energy for 1 second, 11 seconds, 21 seconds and 31 seconds, are observed and recorded. The thermal decomposition properties of two AP samples, which are characterized with the average particle size of $60 \mu \mathrm{m}$ and $6 \mu \mathrm{m}$, respectively, are analyzed by thermogravimetric (TG) analysis and differential scanning calorimetric (DSC) techniques.

\subsection{Calculation of critical decomposition energy}

When AP particles are affected by the electron energy, they will be initiated. The state that the specific sized particle begins to decompose exhibiting with cracks on its surface is defined as the critical decomposition state. Meanwhile, the accepted electron energy is defined as the critical decomposition energy.

(a) Characterization on the critical decomposition state. The S-4800II Field Emission Scanning Electron Microscope (FESEM), whose working voltage and working current are fixed at $15 \mathrm{kV}$ and $10 \mu \mathrm{A}$, respectively, is employed to observe and record the critical decomposition state of the AP particle. The time, that the specific sized particle is affected by the electron energy, is fixed for 3 seconds. When the specific sized AP particle begins to decompose with cracks on its surface under a particular magnification, the critical decomposition state will be captured and recorded by FESEM. If the selected particle doesn't decompose which means no cracks occurring on its surface, then another equal sized particle is selected and affected by the electron energy under a higher magnification. Increase the magnification step by step until cracks occur on the surface of a specific sized AP particle, then capture and record its critical decomposition state. In this way, the magnification, which can affect the projected area, and the accepted electron energy of the AP particle are fixed.

(b) Calculation of the critical decomposition energy. Based on the critical decomposition state of the AP particle shown in the FESEM image, the critical decomposition energy is calculated as the following principle. Firstly, the total electron energy $\left(E_{0}\right)$ affected on the whole FESEM view can be calculated using the working voltage $(U)$, working current $(I)$ and working time $(t)$ as the formula $E_{0}=U \times I \times t$. Secondly, the area of the whole FESEM image $(S)$ and the projected AP particle area $\left(S_{1}\right)$ can be calculated by the Image Pro Plus (IPP) System. Thirdly, the total electron energy are uniformly distributed in the area of the whole FESEM image, and the energy affected on the certain AP particle is in direct proportion to its projected area in the SEM image. Finally, the critical decomposition energy of the specific sized AP particle can be calculated as the formula $\Delta E=E_{0} \times S_{1} / S$.

When the critical decomposition energy is calculated, it is assumed that the affected electron energy is absolutely transformed to be initiation energy. That is, the electron diffraction, the electron transmission, the secondary electron and the characteristic ray won't be considered in this work.

\section{Results and discussion}

\subsection{SEM images of the decomposition course}

Fig. 1 shows the total decomposition course of AP particles under electron energy emitted by the Desktop SEM. The state that the AP particles are affected by electron energy for 1 second without decomposing is shown in Fig. 1a. The state that some of the particles begin to crack, bubble and disappear after 11 seconds is shown in Fig. 1b. The state that most of the AP particles have decomposed to be bubbles and disappeared after 21 seconds is shown in Fig. 1c. And, as shown in Fig. 1d, almost all of the AP particles have decomposed and disappeared after 31 seconds.

As shown in Fig. 1, with the extended time under electron energy, the amount of decomposed AP particles is increased, and the smaller particles begin to decompose earlier. That is, the smaller AP particles are more easily to be initiated.

The corresponding state of AP particles changing from Fig. 1a-d under electron energy is defined as the critical decomposition state. The accepted electron energy in the critical decomposition state is defined as the critical decomposition energy of the specific sized AP particle. The FESEM is employed to observe and record the critical decomposition state of the AP particle and the critical decomposition energy is calculated as shown in the following section.

\subsection{Calculation of critical decomposition energy}

The aforementioned S-4800II FESEM is employed to observe and record the critical decomposition state of a specific sized AP particle. The size of an AP particle is expressed as the diameter of the isovolumetric sphere. The critical decomposition energy is calculated based on the method and the principle shown in "Section 2.3(b)". The critical decomposition energy of the $6 \mu \mathrm{m}$ sized AP particle is selected as the calculation example as below.

As shown in Fig. 2, when the $6 \mu \mathrm{m}$ sized AP particle begins to decompose under electron energy, cracks occur on its surface. Firstly, the electron energy affected on the whole FESEM view can be calculated as the next expression.

$$
E_{0}=U \times I \times t=15 \times 10^{3} \times 10 \times 10^{-6} \times 3=0.45 \mathrm{~J}
$$

Secondly, the effective area of the FESEM image $\left(S_{0}\right)$ and the projected $6 \mu \mathrm{m}$ sized AP particle area $\left(S_{1}\right)$ can be calculated by the IPP System. The areas are expressed in pixels, as shown in the next. 

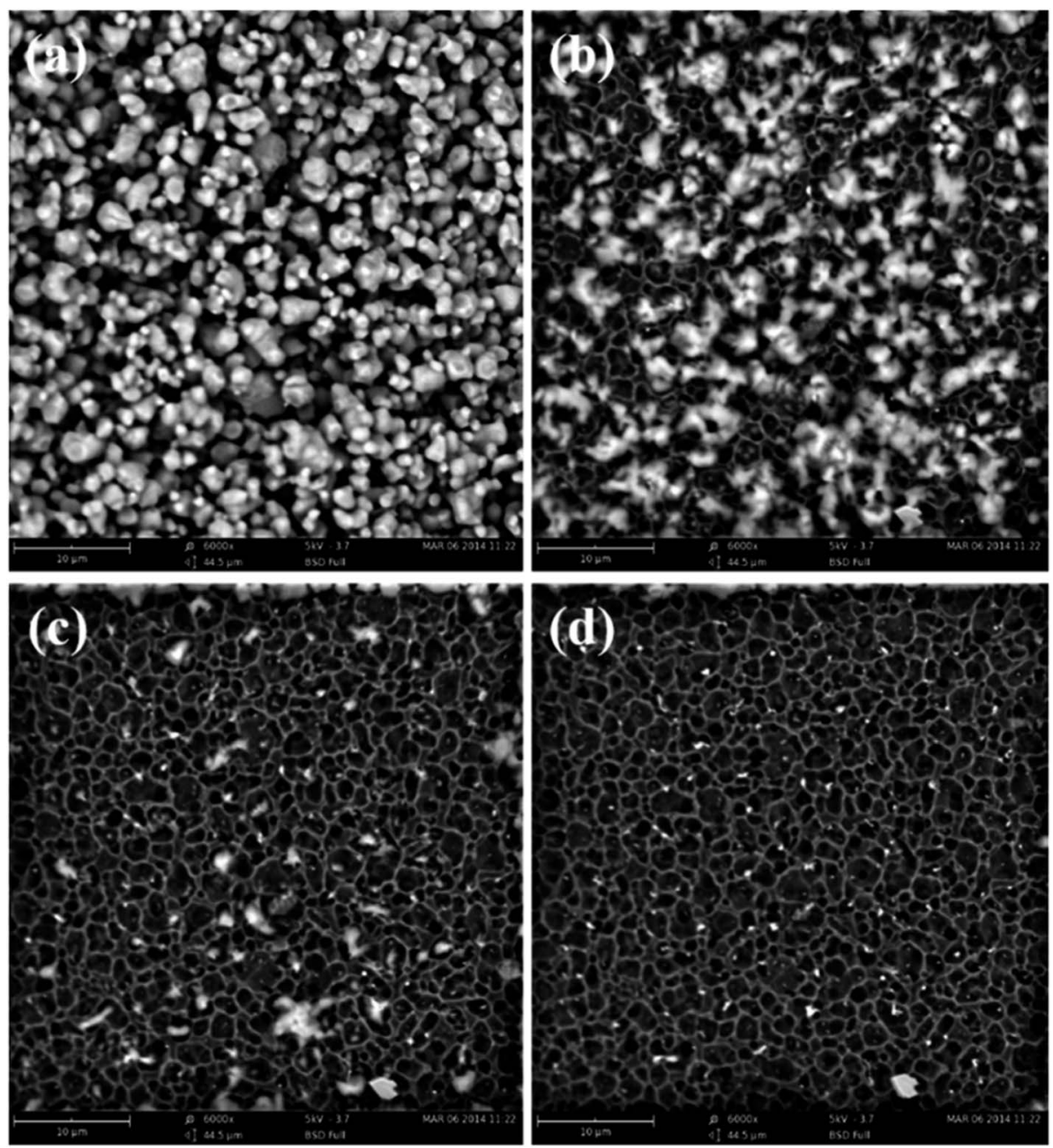

Fig. 1 SEM images of AP particles affected by electron energy for various time: (a) $1 \mathrm{~s}$, (b) $11 \mathrm{~s}$, (c) $21 \mathrm{~s}$ and (d) $31 \mathrm{~s}$.

$$
S_{0}=1137784 ; S_{1}=218907
$$

Then, the critical decomposition energy of the $6 \mu \mathrm{m}$ sized AP particle can be calculated as follows.

$$
\Delta E=E_{0} \times S_{1} / S_{0}=0.45 \times 218907 \div 1137784=0.0866 \mathrm{~J} .
$$

3.3 The changing rule of critical decomposition energy with AP particle size

Based on the method demonstrating in "Section 3.2", the critical decomposition energies of AP particles with different sizes, $1 \mu \mathrm{m}, 6 \mu \mathrm{m}, 10 \mu \mathrm{m}, 20 \mu \mathrm{m}, 60 \mu \mathrm{m}$ and $80 \mu \mathrm{m}$, are calculated and shown in Table 1.
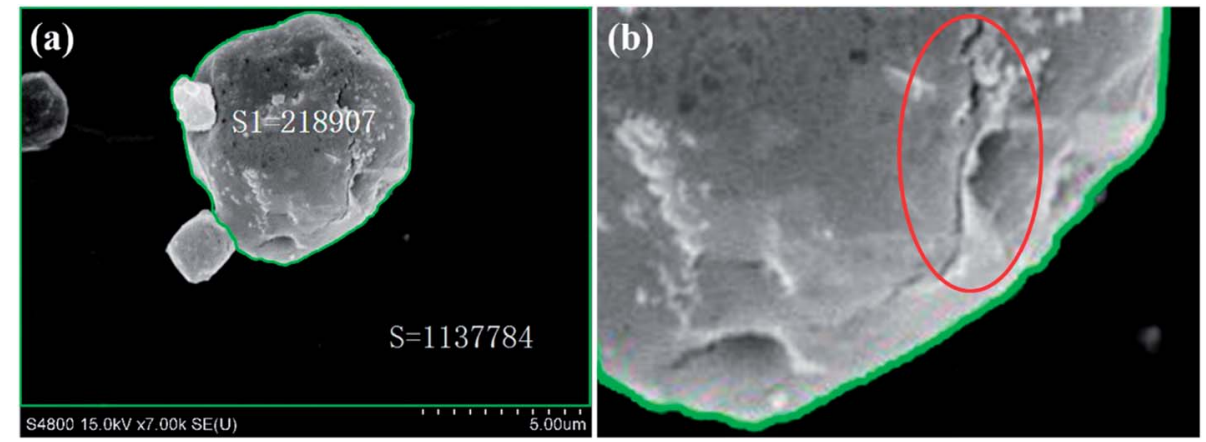

Fig. 2 The critical decomposition state of the $6 \mu \mathrm{m}$ sized AP particle (a) and its detail view (b). 
Table 1 The critical decomposition energies of AP particles

\begin{tabular}{lllllll}
\hline Particle size/ $\mu \mathrm{m}$ & 1 & 6 & 10 & 20 & 60 \\
$S_{0}$ & 1149821 & 1137784 & 1146473 & 1144705 & 1141755 & 1144705 \\
$S_{1}$ & 63170 & 218907 & 372353 & 569218 & 840535 \\
$E_{0} / \mathrm{J}$ & 0.45 & 0.45 & 0.45 & 0.45 & 1040311 \\
$\Delta E / \mathrm{J}$ & 0.0247 & 0.0866 & 0.146 & 0.224 & 0.45 & 0.331
\end{tabular}

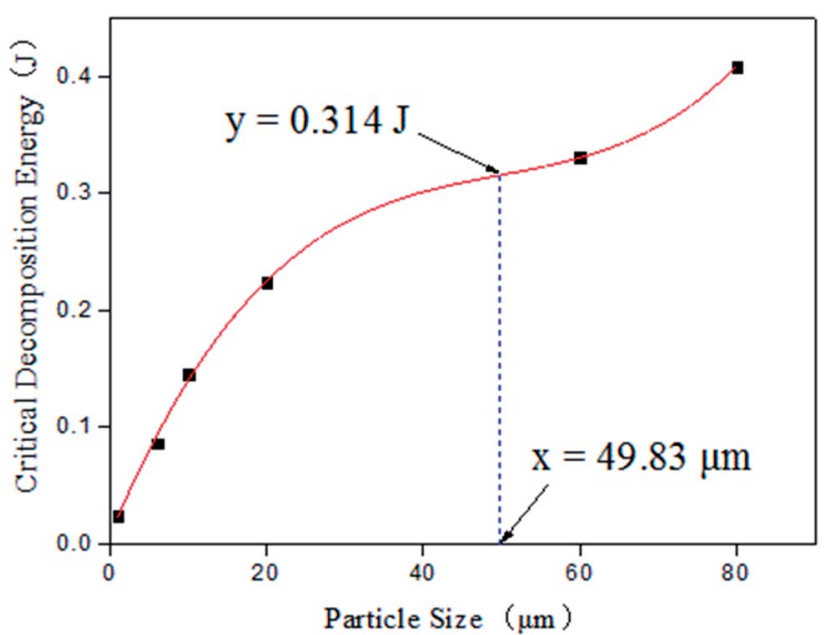

Fig. 3 Relation curve between critical decomposition energy and particle size.

Based on the critical decomposition energy of AP particles as listed in Table 1, the relation curve between critical decomposition energy and particle size is fitted as shown in Fig. 3.
The corresponding function of that curve and its second derivative are shown in formula (1) and formula (2):

$$
\begin{gathered}
y=1.98 \times 10^{-6} x^{3}-2.96 \times 10^{-4} x^{2}+1.60 \times 10^{-2} x+0.00718 \\
y^{\prime}=1.188 \times 10^{-5} x-5.92 \times 10^{-4}
\end{gathered}
$$

As shown in Fig. 3, the critical decomposition energy is decreased with the reduction of AP particle size. It can be known from formula (1) and formula (2) that there is an inflexion point existing in that relation curve, and the inflexion point can be calculated, when the second derivative is equal to zero $\left(y^{\prime}=0\right)$, to be $(y=0.314, x=49.83)$. When AP particle size is above $49.83 \mu \mathrm{m}$, the reduction rate of critical decomposition energy is decreased gradually. When AP particle size is under $49.83 \mu \mathrm{m}$, the reduction rate of critical decomposition energy is decreased rapidly. Especially when AP particle size is approximately less than $20 \mu \mathrm{m}$, the critical decomposition energy is sharply decreased with the reduction of particle size, and the reduction rate is quickly increased. When AP particle size is less than $3 \mu \mathrm{m}$, the critical decomposition energy is very small.
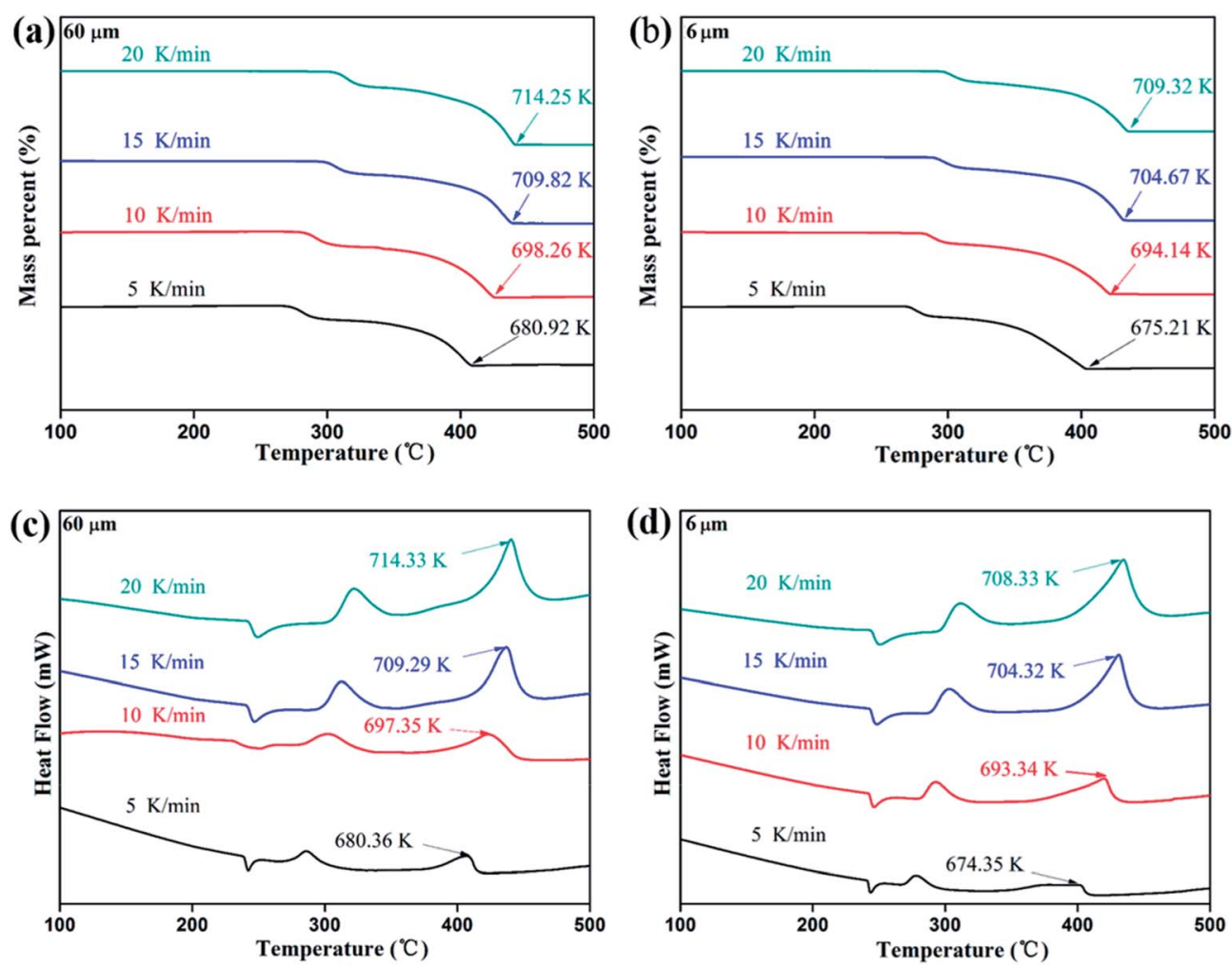

Fig. 4 TG curves (a, b) and DSC curves (c, d) of AP with different particle sizes. 
Table 2 Activation energy of AP with different particle sizes

Activation energy $/ \mathrm{kJ} \mathrm{mol}^{-1}$

$\begin{array}{lllll}\text { Sample } & \text { Kissinger equation } & \text { Starink equation } & \text { Ozawa equation } & {\text { Average value } / \mathrm{kJ} \mathrm{mol}^{-1}}^{2} \\ 60 \mu \mathrm{m} \mathrm{AP} & 148.78 & 148.90 & 152.47 & 150.05 \\ 6 \mu \mathrm{m} \mathrm{AP} & 144.44 & 144.57 & 148.24 & 145.75\end{array}$

To further verify the variation trend of critical decomposing energy with particle size, TG-DSC test are performed at different heating rate and the activation energy were calculated according to Kissinger, ${ }^{13}$ Starink $\mathbf{~}^{\mathbf{1 4}}$ and Ozawa equation, ${ }^{15}$ respectively. The selected AP samples are characterized with the average particle size of $60 \mu \mathrm{m}$ and $6 \mu \mathrm{m}$, respectively, and the TG-DSC curves are shown in Fig. 4.

As can be seen from Fig. 4, the ending temperature and hightemperature decomposition (HTD) temperature are gradually decreased with reduction of particle size. The HTD activation energy of AP samples are calculated from exothermic peak temperature dependence as a function of heating rate shown in Fig. $4 \mathrm{c}$ and $\mathrm{d}$.

As shown in Table 2, the activation energy of the AP sample with the average particle size of $6 \mu \mathrm{m}$ is lower than that of the AP sample with the average particle size of $60 \mu \mathrm{m}$ AP. That is, the AP sample with lower particle size is indicated to be easier to decompose, which is consistent with the variation trend of critical decomposition energy.

Combined with the results of calculated critical decomposition energy and HTD activation energy, it is clearly demonstrated that the superfine AP particles will be easily initiated to decompose by small stimuli. The propellants or FAE are easily initiated to burn or explode, and then the burning rate and brisance of AP-based energetic materials are enhanced. At the same time, their sensitivities to friction, impact and shock are also increased which means the worse safety.

The changing rule between burning rate, brisance or sensitivities of AP based-energetic materials and AP particle size can be explained based on the intuitionisticly calculated critical decomposition energy. If the inflicted energy on AP particles is controlled under the critical decomposition energy, the safety of AP-based energetic materials during development, production, storage, transportation and application will be guaranteed. It is very helpful for the use of superfine AP particles in energetic materials.

\section{Conclusions}

The critical decomposition state of AP particles can be observed and recorded using FESEM, and the critical decomposition energy can be intuitionisticly calculated based on the introduced method. The critical decomposition energy is decreased with the reduction of AP particle size, which is consistent with the variation trend of activation energy calculated from the TGDSC curves. When AP particle size is less than $3 \mu \mathrm{m}$, the critical decomposition energy is very small. The changing rule between burning rate, brisance or sensitivities of AP based-energetic materials and AP particle size can be explained based on the intuitionisticly calculated critical decomposition energy. It is potentially expectable to get the critical decomposition energies of other crystalline materials based on this method.

In future studies, the electron energy loss will be considered. The statistically average critical decomposition energies of AP particles with different size distributions, as well as other crystalline materials, will be calculated and corresponded to the apparent activation energies, so that the energy bridge between microcosm and macrocosm can be built.

\section{Conflicts of interest}

There are no conflicts to declare.

\section{Acknowledgements}

This work was financially sponsored by the National Natural Science Foundation of China (NSFC, 51606102) and the Priority Academic Program Development of Jiangsu Higher Education Institutions (PAPD). Many thanks to Wei-dong Zhou and his group, Yangzhou University, for their helpful discussions in the research area.

\section{References}

1 H. Singh, J. Upadhyay, D. Chimurkar, V. Ghorpade, A. Kotbagi, A. Kumar, A. Kumar and M. Gupta, Propellants, Explos., Pyrotech., 2013, 39, 180-184.

2 Y. F. Liu, Y. Chen, L. Shi and W. S. Yao, J. Energ. Mater., 2014, 32, 71-79.

3 Y. L. Zhu, H. Huang, H. Ren and Q. J. Jiang, J. Energ. Mater., 2014, 32, 16-26.

4 K. Kishore, S. Sankaralingam, R. Nagarajan and K. Mohandas, Def. Sci. J., 2013, 45, 243-254.

5 K. T. Lu, T. M. Yang, J. S. Li and T. F. Yeh, Combust. Sci. Technol., 2012, 184, 2100-2116.

6 C. Franck, F. Yves and E. Charles, Int. J. Energ. Mater. Chem. Propul., 2013, 12, 1-13.

7 X. Han, T. F. Wang, Z. K. Lin, D. L. Han, S. F. Li, F. Q. Zhao and L. Y. Zhang, Def. Sci. J., 2010, 59, 284-293.

8 X. Y. Li, X. L. Liu, Y. Cheng, Y. C. Li and X. L. Mei, J. Therm. Anal. Calorim., 2014, 115, 887-894.

9 S. N. Asthana, C. N. Divekar, R. R. Khare and P. G. Shrotri, Def. Sci. J., 2013, 42, 201-204. 
10 M. K. Choudhari, S. S. Dhar, P. G. Shrotri and H. Singh, Def. Sci. J., 2013, 42, 253-257.

11 M. L. Chan and G. W. Meyers, U.S. Pat., 6955732, 2005.

12 A. A. Borisov, A. A. Sulimov, M. K. Sukoyan, P. V. Komissarov, I. O. Shamshin, R. Kh Ibragimov and Yu. M. Mikhailov, Russ. J. Phys. Chem. B, 2009, 3, 936-944.
13 T. Chen, P. Du, W. Jiang, J. Liu, G. Z. Hao, H. Gao, L. Xiao, X. Ke, F. Q. Zhao and C. L. Xuan, RSC Adv., 2016, 6, 8383883847.

14 M. J. Starink, J. Mater. Sci., 1997, 32, 4061-4070.

15 Y. Cheng, Y. Li, S. Yan and C. Huang, J. Math. Chem., 2010, 48, 704-713. 